\title{
CHARPENTIER, « LIGUEUR » Y AUTOR DE LA PRIMERA GRAMÁTICA DEL ESPAÑOL PARA FRANCESES
}

\author{
Marie-Hélène Maux-Piovano \\ Université de Strasbourg. Culture et Histoire dans l'Espace Roman (CHER. EA 4376) \\ pievanus@wanadoo.fr
}

\section{La identidad del autor de La Parfaicte Methode}

Han llegado hasta nosotros dos ediciones de La Parfaicte Methode pour entendre, escrire, et parler la Langue espagnole. La primera lleva la fecha de 1596, y dos ejemplares de ésta se encuentran la Biblioteca Nacional de Francia, pero no son idénticos y existen algunas diferencias entre ambos (Quijada, 2006: 323). La segunda, de 1597, está ubicada en la Biblioteca Nacional de Madrid ${ }^{1}$. Esta gramática, cuyo objetivo es enseñar el español a los franceses desde una perspectiva contrastiva teniendo en cuenta las características propias de los destinatarios, es la primera de una serie de manuales que unos doce autores publicarían en Francia a lo largo del siglo XVII, hasta 1661, aprovechándose de los acontecimientos políticos y del interés que éstos podían provocar por la lengua española ${ }^{2}$. Ahora bien, mientras que los demás autores del corpus quedan perfectamente identificados, conociéndose, al menos aproximadamente, su biografía, La Parfaicte Methode... quedó anónima hasta 1901, cuando el hispanista A. Morel-Fatio descubrió en el ejemplar RES-X-2077 de lo que era en aquel entonces la Biblioteca Nacional de París una nota manuscrita que indicaba que aquella gramática habría sido redactada

Par N. Charpentier, filz de Carpentarius lecteur du Roy, ennemi de Ramus. Ce Charpentier icy fut roué à Paris tout vif au mois d'avril 1597 pour une entreprise sur Rouen et autres villes maritimes [faiste] par les Espagnols, a [la suite de (?)] la sollicitation du deffunt evesque de Nantes, lors evesque de St Malo; il s'y employa ${ }^{3}$.

El hispanista francés estableció una relación entre estas afirmaciones y la aserción que hacía en 1615 Ambrosio de Salazar en su Response apologetique au libelle d'un nommé Oudin; en efecto, el maestro de español evocaba «celui qui fut roué en place publique pour

1 Las signaturas de las ediciones de la BNF son RES-X-2077 et RES-X-2592, como ya precisa A. Morel-Fatio en 1901 (pero no copia la mención RES), y la de la edición de Madrid a la que nos referimos es R-13477. Esta tiene un error en el orden de los folios del primer cuaderno; lo señalaremos cuando nos refiramos a ella.

2 Véase Maux-Piovano, Marie-Hélène (2002), Les débuts de la didactique de l'espagnol en France : les premières grammaires pratiques (1596-1660), Lille, Presses Universitaires du Septentrion.

3 Hemos añadido entre corchetes las palabras que resultan de nuestra lectura personal y que faltan en la de MorelFatio. El segundo corchete corresponde a un fragmento de difícil lectura. 
avoir enseigné la langue espagnole», para citarle a Oudin una gramática del español escrita por un francés de una calidad muy superior a la Grammaire et observations de la langue Espagnolle recueillies \& mises en François ${ }^{4}$ que el Secretario e Intérprete de Enrique IV andaba reeditando desde 1597. Morel-Fatio cotejó estos datos con los Mémoires-journaux de Pierre de l'Estoile y estableció que el autor de la primera gramática española para franceses era «N. Charpentier», hijo de Jacques Charpentier, «lecteur et medecin en l’Université de Paris», tristemente famoso por haber participado activamente en la noche de San Bartolomeo en 1572, durante la cual habría provocado el asesinato del filósofo Pierre de la Ramée. En adelante, $L a$ Parfaicte Methode... ya no era anónima, su autor tenía apellido aunque todavía no llevaba nombre de pila. Consideramos como convincente la demostración del estudioso del siglo pasado, y nos parece que el hecho de que coincidan el testimonio de Salazar, contempóraneo de Charpentier, una nota manuscrita (aunque todavía no hemos identificado la época de su redacción) y una crónica del siglo XVII no deja ninguna duda a la hora de afirmar que el autor de La Parfaicte Methode... es un llamado «Charpentier» que todos están de acuerdo en considerar como el hijo de Jacques, famoso en la universidad de París en el siglo XVI. Falta pues descubrir cuál fue su nombre, que en los documentos consultados por Morel-Fatio sólo aparece bajo la forma de una letra inicial en la nota manuscrita de la edición de París.

Durante unas investigaciones anteriores nos había llamado la atención esta letra inicial que se parece a una $N$ pero, como dijimos en otras ocasiones, se interpretó a veces como una $M$ (Sánchez Pérez, 1992, López y Seré 2000). La grafía $N$, que nos parece más probable, suscitó la atribución a Charpentier de diferentes nombres cuya fuente nunca se indica, como Nicolas (Vanderlynden, 1984) o Noël, nombre que también hemos utilizado a veces. Dado que el presente artículo quisiera proporcionar algunos elementos complementarios sobre la identidad y la vida de Charpentier, hemos tratado primero de arrojar luz sobre su nombre.

Para empezar hemos consultado los diccionarios de biografía más famosos, entre los cuales sólo el Dictionnaire de biographie française de Roman d'Amat alude a la participación de Charpentier en la Liga católica, este movimiento ultracatólico, polimorfo, que pretendía eradicar el protestantismo de Francia con la ayuda de España y de los Jesuitas. Los datos que proporciona Roman d'Amat resultan bastante precisos ya que en el artículo que parece referirse al autor de la gramática española le da un nombre a Charpentier, que se habría llamado Antoine, y precisa que era un miembro muy activo de la Liga. Fue esta referencia la que utilizamos en nuestra tesis de doctorado para justificar que le atribuíamos el nombre Antoine a Charpentier. Gracias a Roman d'Amat, supimos que además que se casó con la hija de Du Bacquet, que desempeñaba el cargo de «avocat du roi à la Chambre du Trésor». La fuente principal de esta noticia biográfica es un artículo que se publicó con el título «Un conspirateur à Chennevières-sur-Marne», en un boletín local titulado Le Vieux Saint-Maur, en 1924 y su consulta nos permitió añadir información a lo que expone Roman d'Amat. El autor de dicho artículo firma «Casenave, Ministre plénipotentiaire, Ancien Conseiller municipal de Chennevières-sur-Marne», sin dar más precisiones. Casenave llama al conspirador evocado en el título "Antoine Charpentier», y el artículo se publicó con el motivo de que a finales del siglo XVI habría sido Antoine Charpentier propietario de una granja y de un

4 La primera edición es Grammaire et observations de la langue Espagnolle recueillies \& mises en François, Paris, chez Marc Orry, ruë St Jacques, au Lyon Rampant. La biblioteca de Estrasburgo (BNUS) posee un ejemplar cuya signatura es Cd 122406 . 
parque en Chennevières. Con este pretexto, Casenave presenta la vida del padre de Antoine, Jacques, y a continuación algunos elementos de la de Antoine. Está claro que utiliza las memorias de Pierre de l'Estoile, pero se vale también de otras fuentes que desgraciadamente no hemos podido identificar y que le permiten transcribir en estilo directo las palabras del Duque de Mercoeur y de Du Plessis-Mornay. Dado que Le Vieux Saint-Maur no era una revista científica no cuenta con ninguna bibliografía, lo que nos impidió entender por qué Casenave le atribuye el nombre de Antoine al hijo de Jacques, ya que sabemos que no pudo sacar esta información de de L'Estoile.

Para buscar la pista de Charpentier empezamos pues interesándonos por los miembros de la Liga que pasaron a la historia y particularmente por los que participaron en el llamado «Conseil des Seize» de la ciudad de París. En 1596 ya está oficialmente disuelta la Liga católica. En efecto, Enrique IV había entrado en París el 22 de marzo de 1594, y las tropas españolas que ocupaban la capital francesa empezaron a marcharse ese mismo día. Por lo tanto, Charpentier perteneció a la minoría de «ligueurs», muy poco numerosos, que siguió actuando a escondidas después de la disolución de la Liga. Esta constatación fue lo que nos llevó a referirnos a unos estudios históricos que tratan de la identificación de los miembros de la Liga parisiense, tanto desde un punto de vista biográfico como social y político. Nos fueron de mucho provecho la publicación de Elie Barnavi Le parti de Dieu. Etude sociale et politique des chefs de la Ligue parisienne 1585-1594, y la de Robert Descimon Qui étaient les Seize?, aunque encontramos en ésta menos información que en Barnavi. Barnavi incluye a Charpentier entre los cabecillas de la Liga, y conforme desarrolla sus análisis le proporciona al lector unos datos a propósito de su vida social y de las vicisitudes de su existencia cuando empieza a declinar la Liga. Descimon, en el capítulo central de su libro, sintetiza en una serie de fichas clasificadas alfabéticamente todas las informaciones que recopiló a propósito de los miembros de la Liga. Estos dos estudios se refieren casi exclusivamente al Archivo Nacional de Francia, y al «minutier central des notaires de Paris», que es un elenco de las actas antiguas firmadas en los bufetes de los notarios parisienses. Los dos investigadores le atribuyen sin vacilar el nombre de Antoine a Charpentier, ya que es el que aparece en las actas notariales y Descimon precisa en efecto que era hijo de Jacques, «lecteur du roi, docteur régent en la Faculté de Médecine de Paris» ${ }^{5}$. Barnavi lo incluye dentro de la categoría social de los «nobles hommes et maîtres» y su profesión era la de abogado. Según Descimon vivía en la calle des Vieilles Estuves, en la parroquia de Saint-Eustache. El «minutier central des notaires parisiens» les permitió a los dos historiadores establecer que Antoine Charpentier se casó el 28 de agosto de $1588^{6}$ con Madeleine Bacquet, hija de Jean Bacquet, a quien se incluye también en la categoría de los «nobles hommes et maîtres», como su nuero, y quien era abogado y «conseiller du roi au trésor». Barnavi cita esta boda como ejemplo de la ascención social que lograban algunos cabecillas de la Liga casándose. En el caso de Charpentier, lo que prueba esta ascención son la cantidad de la dote y la respectabilidad de los testigos de la esposa.

5 Es evidente que no existe ningún vínculo entre Antoine y la ficha siguiente que se refiere a un tal François Charpentier, «marchand de vin et de poissons de mer». Es un homónimo.

6 Según Descimon, el 28 de agosto de 1588 es la fecha en la que se firmaron las capitulaciones, lo que no impide que fuera también la fecha de la boda. Barnavi precisa que la dote consistía en «3000 écus soleil en espèces, le gîte et le couvert du jeune couple avec un serviteur et une servante [...]» (Barnavi, 1980: 103). 
También gracias a las investigaciones de Barnavi hemos encontrado algunas huellas de Antoine entre su casamiento y su muerte trágica. En 1591, el Duque de Mayenne, jefe de la Liga Parisiense vuelve a París y se disuelve de facto el «Conseil des Seize», ya que se ajustician sus miembros más activos. En este contexto, se menciona a Charpentier como uno de los ocho abogados que quedan «dans le groupe de chefs ligueurs en ces temps de vaches maigres» (Barnavi, 1980: 215). En 1589 eran quince.

En diciembre de 1591 pues, Charpentier se encuentra en París. Se vuelve a encontrar su huella en 1594: después de la conversión de Enrique IV y de su entrada en París, el Parlamento promulga el 28 de marzo un fallo que condena la Liga y empiezan a circular unas listas de proscritos. A pesar del perdón del rey que, por lo menos, les salva la vida, se incita a los «Seize» a que se destierren, alejándose de París o incluso afincándose en el extranjero. La lista de los proscritos consta de ciento dieciocho nombres, entre los cuales cuatro abogados, pero el de Charpentier no aparece. Barnavi recopila los nombres de cuarenta y un cabecillas de la Liga que se encontraban en París en aquella época, y establece que treinta y siete de ellos se exiliaron, pero otra vez más Charpentier constituye una excepción, ya que es uno de los cuatro que se queda en la capital. ¿Sería particularmente astuto? ¿Se habría beneficiado de la protección de poderosos personajes? Lo cierto es que debió de intentar vivir en la mayor discreción posible, y quisiéramos imaginar que se aprovechó de este ocio forzoso para escribir su manual de lengua española. Sin embargo, como vamos a mostrar a continuación, no se dedicó sólo a estudiar en aquel periodo.

\section{Antoine Charpentier y su relación con España}

Después de establecer la participación en la Liga y, por lo tanto, las simpatías pro españolas de Antoine Charpentier, hemos intentado indagar más a propósito de los acontecimientos que lo llevaron a morir ajusticiado en la famosa Place de Grève. Para eso hemos consultado los memorialistas y los cronistas de los siglos XVI y XVII, y hemos encontrado varios relatos de la detención y del ajusticiamiento de Antoine, con algunas variantes. Hemos hallado datos interesantes en la Histoire Universelle de Jacques-Auguste de Thou, en el Journal du regne de Henry IV Roi de France et de Navarre de Pierre de l'Etoile en la edición aumentada por las notas del «Chevalier $\mathrm{CBA}^{7} »$ y finalmente, aunque el autor es más sintético, en la Chronologie novennaire de Palma Cayet. Cabe reconocer que el nombre de Charpentier sólo se menciona en estas crónicas a propósito de los acontecimientos del año 1597, fecha de su muerte, aunque en una de sus glosas del Journal de Pierre de l'Estoile, el chevalier CBA precisa en una nota que Charpentier era el «Chef d'un petit Conseil de Ligueurs, qui étoit à Paris entretenu par lui et par l'argent d'Espagne». (De l'Estoile, 1741: 350). Desgraciadamente no hemos podido identificar a qué consejo aludía el comentarista.

Los tres memorialistas confirman que Charpentier era abogado y de Thou precisa que ejercía su profesión en París. Pierre de l'Estoile y Jacques-Auguste de Thou añaden que era hijo de Jacques Charpentier, e insisten un poco en la personalidad del padre: lo presentan como un ultracatólico que desempeñó un papel muy activo durante le noche de San Bartolomeo y lo designan como el instigador del asesinato de Ramus contra el cual quería saciar

7 Según el Dictionnaire des ouvrages anonymes et pseudonymes, de Antoine-Alexandre Barbier, se trataría del abad Lenglet du Fresnoy que se hubiera valido de este seudónimo para mantener el anonimato. 
una venganza personal ${ }^{8}$. Es cierto que nuestro hispanista proviene de una familia de letrados, pero cuyos miembros no tenían ningún escrúpulo y eran unos antiprotestantes convencidos.

La fuente más completa y más fiable es sin duda alguna la Histoire Universelle de JacqueAuguste de Thou, dado que el autor fue testigo directo de los acontecimientos que siguieron a la toma de Amiens por los Españoles, habiéndole encargado el rey que negociara con los diputados protestantes y con el duque de Mercoeur, junto con Gaspard de Schomberg, conde de Nanteüil (De Thou, 1734: 128). Es evidente que para De Thou la historia de Charpentier forma parte de los últimos sucesos de la historia de la Liga, ya que la designa en el índice del libro CXVIII con el título «Intelligences du Duc [de Mercoeur] avec les Espagnols découvertes par quelques lettres interceptées» (De Thou, 1734: IV). La detención del hispanista-hispanófilo, que confabulaba contra el gobierno que acababa de establecer Enrique IV, depende estrechamente del devenir de tres series de cartas que hubieran debido permanecer secretas.

La tres fuentes están de acuerdo en afirmar que la suerte de Charpentier estaba vinculada con la de otro personaje, llamado Des Losges, a propósito del que de L'Estoile sólo menciona que era «courrier» (o sea, mensajero, ya que llevaba mensajes), pero que Palma Cayet presenta como «un jeune advocat de Beauvais». Es evidente que Des Losges tenía una relación con Charpentier, y si confiamos en el «Chevalier CBA» incluso era su sobrino (De L'Estoile, 1741: 350). Según de Thou (1734: 131), era «un jeune homme de Beauvais, nommé La Croix, dévoüé à la Ligue, \& qui résidait en Flandres». En una nota, el memorialista precisa que La Croix era un seudónimo y que la relación entre Des Losges y Beauvais era que su padre había nacido en aquella ciudad. Sin embargo, nos hemos acordado de que Jacques Charpentier, el padre de Antoine, había nacido en Clermont-en-Beauvaisis en 1521. Quizás se trate de una mera casualidad, pero en el artículo del Bulletin du Vieux Saint-Maur que ya hemos evocado, Casenave subraya que en la misma época en la que Charpentier poseía una granja y un parque en Chennevières, una familia de apellido Des Losges tenía una propiedad en la misma localidad. Para Casenave, se trataría de «une coïncidence curieuse» (Casenave, 1924: 46). Sugerimos que estas «coincidencias» podrían explicarse por los lazos de sangre entre las dos familias. En cambio, consideramos como imposible que nuestro Des Losges se identifique con el llamado Jehan Deslosges, muerto en 1596, cuya ficha biográfica recopila Descimon (Descimon, 1983: 132) .

Bien parece que el gramático fuera el instigador de todo el asunto mientras que Des Losges sólo ejecutaba órdenes. Le habían encargado que informara al Duque de Mercoeur de

8 Tuvo una pendencia con el filósofo porque éste nunca había aceptado que comprara una cátedra de matemáticas en el Collège de France gracias a la protección de la familia Guise mientras que él mismo reconocía que no sabía nada de matemáticas. Jules Michelet, en el tomo VIII de sus obras completas, trata detenidamente de Jacques Charpentier, de su personalidad atormentada y de su gusto por la intriga, así como de sus esfuerzos para alejar las teorías copernicanas de la universidad y de su vil venganza contra Ramus, al que había hecho perder su cátedra en la universidad al firmarse la paz de 1570. El historiador recuerda que J. Charpentier nunca se arrepintió de su participación en la noche de San Bartolomeo, que designaba como «ce brillant, ce doux soleil qui a éclairé la France au mois d'août » (Michelet, 1980: 258).

9 Jehan Deslosges ejercía la profesión de mercero y había tenido un momento de fama trágica durante la noche de San Bartolomeo. Además, había asesinado a un inglés (véase Palma Cayet), y murió de mala muerte en 1596 porque se derrumbó el puente en el que se encontraba, que era el mismo desde el que había despeñado a su víctima. Por otra parte, Barnavi escribe el nombre del mercero en una palabra sola mientras que el nombre del cómplice de Charpentier aparece en dos palabras. Casenave piensa que se puede afirmar que el cómplice de Charpentier era el hijo del mercero, pero nada justifica esta filiación. 
la buena noticia de la toma de Amiens por los Españoles. De Thou dice que «il était chargé d'ordres secrets, et de quelques lettres du Cardinal Albert ${ }^{10} \gg$ (De Thou, 1734: 131). Una mera circunstancia material en Saumur provocaría su ruina: el caso era que sólo el gobernador del lugar podía autorizar que se entregaran caballos frescos a los viajeros, y fue lo que provocó el encuentro casual entre Philippe du Plessis Mornay y Des Losges, y por consiguiente la detención de éste porque Du Plessis juzgó que era sospechoso. Encontraron unas cartas escondidas en el establo que, aunque estaban parcialmente cifradas, demostraban sin duda alguna que el Duque de Mercoeur tenía acuerdos con España y que la Liga todavía se beneficiaba de cierta potencia militar. Dos de las cartas las firmaba el Archiduque Alberto y se dirigían respectivamente al Duque de Mercoeur y a Rodrigo Mendone ${ }^{11}$, el representante de Felipe II en Bretaña. Palma Cayet precisa que el Archiduque le pide a Mercoeur que haga la guerra en Bretaña y que el duque de Savoya la haga en el Delfinado para dividir las tropas reales y conservar el dominio de Amiens (Palma Cayet, 1606: 760). No cabe pues ninguna duda de que existía una coalición contra Enrique IV: el gobernador de los Países Bajos le da al Duque de Mercoeur la palabra de España. Además, en la parte cifrada de una carta, se expresa la amenaza de mandar un «ejército auxiliar», que llegaría de España poco tiempo después y se sugiere que pronto vencería la Liga, cosa perfectamente inverosímil en 1597. Se consideraron los hechos como asunto de estado y por consiguiente se convocó a Des Losges para que se explicara ante el rey. Las declaraciones de Des Losges darían lugar a un segundo asunto que llevaría al autor de La Parfaicte Methode a su ruina.

En efecto, el duque de Mercoeur, que desempeña un papel fundamental, no era sólo destinatario: también era el remitente de otra serie de cartas dirigidas a «Charpentier Avocat au Parlement de Paris, fils du célébre [sic] Jacque [sic] Charpentier, Professeur de Philosophie dans l'Université de Paris» (De Thou, 1734: 133). Si se admite que Charpentier y Des Losges tenían lazos de familia, quizás se pueda entender por qué el Duque de Mercoeur se preocupó tanto por salvarle la vida; designándolo con el nombre de La Croix ${ }^{12}$, le propone a Charpentier declarar que este mensajero era un criado del Archiduque de Austria y ofrece el dinero necesario para el rescate. Esta carta lleva la fecha del 8 de abril 1597. Pero lleva también otras noticias, mucho más políticas, ya que vuelve a evocar los supuestos refuerzos militares españoles y la invasión de Francia por Alberto, lo que pondría a Enrique IV en una situación peligrosa. Se van precisando las amenazas en la continuación de la carta, mencionando Mercoeur que se le ha mandado un emisario a Felipe II para solicitar armas y dinero; además, Mercoeur propone ir personalmente a los Países Bajos para ponerse a las órdenes de Alberto. Considerando sin embargo que este proyecto es muy difícil de realizar, propone que se invada Francia a finales de julio, al acabarse la tregua. Siendo Charpentier el destinatario

10 El Cardenal Archiduque Alberto fue nombrado gobernador general de los Países Bajos por Felipe II. Era hijo de Maximiliano de Austria y se casó en 1599 con Isabel Clara Eugenia, hija de Felipe II, o sea, de su primo hermano.

11 De Thou precisa que Rodrigue Mendone estaba «chargé des affaires de Philippe dans la Bretagne».

12 En la edición de 1741 del Journal de Pierre de l'Estoile, el «chevalier CBA» cita además el nombre de Crucé, que sería como Philippe Mornay du Plessis designaba a Des Losges alternando con «le Journaliste Des Losges». Se podría establecer una relación entre el seudónimo de Des Losges, La Croix, y el nombre utilizado en las glosas, Crucé, ya que parece la forma latina levemente modificada. Sin embargo, uno de los miembros del «Conseil des Seize» se llamaba Oudin Crucé. Este Oudin Crucé se refugiaría en los Países Bajos. Aunque sólo el «Chevalier CBA» evoca el nombre de Crucé, no excluimos que Des Losges se haya valido del nombre de un «ligueur» famoso, quizás para esconderse. 
de esta correspondencia, se encuentra en una situación muy delicada, por no decir peligrosa. De Thou no da ninguna precisión a propósito de las circunstancias del descubrimiento de esta serie de cartas.

Una tercera serie conduciría a la detención del gramático (De Thou, 1734: 134): su autor es un tal Valet, al que de Thou designa como el prior de la Trinité, en Bretaña. Aunque se había disimulado el nombre del destinatario, sustituyéndolo por un seudónimo, el biógrafo Michel Prévost piensa que se trata del Chancelier Hurault de Cheverny (Roman d'Amat, 1959: 630). El «prévôt de la Connétablie» Nicolas Rapin, que ya instruía el caso Des Losges, mostró que el remitente era Charpentier. Según Prévost, lo denunció Des Losges porque lo atormentaron, lo que le quita algo de mérito a la «sagacidad de Rapin» que alaba de Thou. Después de esta denuncia, se detuvo a Charpentier y se organizó una confrontación con su sobrino. Se descubrió que Charpentier desempeñaba un papel muy importante en lo que quedaba de la Liga, ya que era el correspondiente del Archiduque Alberto para quien hacía de intermediario haciendo llegar sus cartas hasta los últimos partidarios de la Liga, particularmente en Bretaña donde eran todavía numerosos.

Se condenó al tío y al sobrino al tormento de la rueda por alta traición. Los ajusticiaron el 10 de abril de 1597 (De l'Estoile, 1741: 348), después de pasearlos desde el Grand Châtelet hasta la plaza de Grève «avec des escriteaux pendus à leur col» precisa Palma Cayet (Palma Cayet, 1606: 760). Sin embargo los dos condenados no renegaron de sus convicciones y ambos denunciaron la situación en la que se hallaba su país. Pero mientras que Charpentier murió dignamente, contentándose con algunas consideraciones generales, Des Losges habló mucho y pareció cobarde.

Así fue cómo murió el primer autor de una gramática del español para franceses ${ }^{13}$, y esta muerte prematura debió de ser lo que le impidió que redactara la segunda parte de su método, que hubiera debido incluir «les recherches des plus beaux enrichissements de la langue qui servent à la composition et traduction». Lo lamentamos por todo lo que hubiera podido traer a la historia de los principios de la enseñanza del español en Francia, ya que sus competencias como lingüista y como hispanista no dejan lugar a dudas y destacan regularmente en la primera parte de su método como lo vamos a mostrar a continuación, aunque cabe subrayar que en ninguna crónica se alude a la actividad de gramático de Antoine Charpentier.

\section{EI hispanista en su método}

No vamos a exponer aquí las numerosas pruebas que da Charpentier de su cultura literaria española que traslucen regularmente en su gramática: ilustra las reglas que enuncia con unos ejemplos sacados, según sus propias palabras, de los «plus doctes Autheurs» (Charpentier, 1597: f. 63 v), o sea, esencialmente, Alonso de Ercilla, Juan de Mena, Boscán, Fray Antonio de Guevara y Garcilaso de la Vega. Aunque destaca su cultura literaria sobre todo en comparación con la de la mayoría de sus colegas (ligeramente posteriores), estas citas no bastan para indicar que tenía una práctica oral de la lengua española que le diera una verdadera competencia de comunicación.

13 De l'Estoile precisa a finales del mes de abril de 1597 que la muerte del nuero provocó la del suegro, Jean de Bacquet, quien no aguantó este final infamante (De l'Estoile, 1734: 354).

14 Además de las gramáticas de Nebrija y Miranda, naturalmente. 
Cabe considerar de manera diferente la utilización de los refranes que, aunque se pueden incluir en el campo de lo escrito, puesto que los refraneros constituyen un subgénero literario, están estrechamente vinculados con el conocimiento enciclopédico de la lengua, particularmente cuando se trata de evaluar de modo pertinente el contexto de enunciación. Ahora bien, apuntamos diecisiete refranes o expresiones proverbiales en la gramática de Charpentier, sin que se haga referencia a ningún refranero. Proponemos ver aquí un primer indicio del conocimiento íntimo del español en un contexto de comunicación oral, en una época y en una sociedad en las que los refranes tienen «une place à part entière dans le discours» (Chevalier, 1988: 72). Además, en relación con seis de ellos, Charpentier precisa que son refranes «castellanos» ${ }^{15}$, lo que se opone a la simple indicación de «refrán» o a la ausencia de indicación que asimila el refrán a un ejemplo cualquiera. Ahora bien, muchos indicios en la exposición gramatical muestran que para Charpentier «castellano» no es sinónimo de «español». Por ejemplo cuando evoca a los locutores de las diversas lenguas europeas, opone los italianos, los franceses etc... a los españoles, y no a los castellanos. Parece que éstos ocupan un lugar particular en su sistema de referencias y que este defensor de la fe católica, letrado, hispanófilo e hispanófono, cuya vida ignoramos casi por completo, hubiera tenido la oportunidad de viajar por Castilla o, al menos, que hubiera mantenido intercambios lingüísticos suficientemente provechosos con nativos como para sacar conclusiones notablemente pertinentes en cuanto al uso de la lengua hablada.

Advierte por ejemplo al lector que «les Castillans appellent un oyseau sanchico qui est naturellement babillard [...]» (Charpentier, 1597: f. 10v et 11r). Pero también ha notado algunas realizaciones fonéticas propias de los «villageois de Castille», así como la tendencia a sustituir los [f] y [h] iniciales por un [g] : «On pourrait encore excepter guevo, guente, pour huevo, fuente, n'estoit que c'est plustost un dialecte et façon de parler de quelque villageois de Castille que la vraye orthographe.» (Charpentier, 1597: f. 7 v, paginación errónea.) Subraya también la confusión entre el [r] y el [1] después de una oclusiva «[...] en beaucoup d'endroits de Castille, $r$ se change en $l$. cabrito, cablito, gruesso, gluesso, gritador, glitador, ce qui provient de certaine débilité de langue.» (Charpentier, 1597: f. 8 r., paginación errónea).

En la parte dedicada a la descripción fonética, que titula «Des Lettres», es donde Charpentier deja ver involuntariamente que tenía una verdadera práctica de la lengua española, además de sus conocimientos librescos. Conforme con la costumbre de la época, sigue los modelos de los tratados de pronunciación más famosos en el siglo XVI, y todo sugiere que se inspira directamente en las Osservationi de Miranda. Para el grafema B, esta imitación lo lleva a proponer una analogía entre las realizaciones fonéticas francesa, latina y española. Pero el sutil observador de la lengua que debía de ser Charpentier no se satisface con esas descripciones teóricas que sólo repiten lo que dicen fuentes antiguas sin tomar ninguna distancia crítica. De este modo, indica que en Castilla, y «spécialement par tout le Diocese de Burgos», los grafemas B y V tienen una realización fonética única, lo que puede acarrear confusiones gráficas. Afirma pues que B se pronuncia como «v consonante», lo que nos indica de

15 Se trata de Arrendardocillo, comer en plata morir en grillo, Que nobleza de señor no nos conoce y enbianos a saludar, Guarte de puta que dexa la bolsa enxuta, El comer y il (sic) rascar todo \& (sic) comenzar, entrar lamiendo, salir mordiendo y La que del baño viene bien sabe lo que quiere. El penúltimo es el único que no recopila Correas. 
modo cierto que ha observado puntualmente la reducción a un fonema único y puede incluso sugerir que tenía conciencia de la fricatización.

En cuanto al grafema Ç (o C delante de I o E), el gramático se contradice abiertamente al proponer una descripción articulatoria. Expone en primer lugar el resultado de sus observaciones personales: «[le Ç] se prononce avec un doux sifflement en mettant le bout de la langue entre les dents de devant» (Charpentier, 1597: f 4 v). Parece evidente que está describiendo la interdental /e/, lo que es bastante precoz si se considera la evolución del sistema fonético del español, pero perfectamente plausible si admitimos que se refiere a Castilla y más precisamente a la región de Burgos. Ahora bien, el final de la frase contradice esta proposición innovadora ya que añade «et lors il ha la mesme force que le $z$ des Italiens». Dicho de otra manera, está describiendo la africada medieval /ts/. Se impone de nuevo la autoridad del escrito, y se puede temer que esto precisamente fue lo que le impidió al gramático sacar más provecho de sus observaciones personales.

Cabe reconocer que los elementos que hemos podido entresacar para mostrar que Charpentier tenía una práctica del español oral no son muy numerosos: nos hubiera gustado afirmar que hacía de intérprete entre españoles y franceses durante su participación en la Liga Católica; desgraciadamente en este sentido nuestras investigaciones han sido infructuosas. Sin embargo, nos parece que los pocos elementos que entresacamos corroboran lo que sugerimos de la personalidad del gramático, observador de la lengua atento y culto.

\section{Conclusiones}

La exposición de los resultados de estas investigaciones en las crónicas del siglo XVII y en los estudios de los historiadores actuales que se centran en la Liga debería permitir ante todo que se le devolviera su nombre al primer gramático del español que se dirigió estrictamente a franceses, más de un siglo después de que se le restituyera su apellido. Se precisan asimismo los conocimientos biográficos sobre la personalidad compleja de Charpentier, personaje tan culto como intolerante. Los elementos biográficos que se han entresacado subrayan la importancia de sus vínculos con España, y parecen tan estrechos que traslucen en su gramática a través de una serie de indicios pragmáticos y lingüísticos que se han intentado sacar a luz.

\section{Referencias bibliográficas}

Barbier, A-A. (1823): Dictionnaire des ouvrages anonymes et pseudonymes. Tome second, Paris, chez Barrois l'aîné.

Barnavi, E. (1980): Le parti de Dieu. Etude sociale et politique des chefs de la Ligue parisienne 15851594. Louvain, Nauwlaerts.

Casenave, (1924): «Un conspirateur à Chennevières-sur-Marne». En Le Vieux Saint-Maur, Bulletin. Paris, págs. 43-50.

Cayet, P-V., dicho Palma (1606): Chronologie novennaire, histoire des guerres de Henri IV de 1589 à 1598. Paris.

Charpentier, N. (?) (1597): La parfaicte methode pour entendre, escrire et parler la langue Espagnole divisée en deux parties [....]. Paris, chez Lucas Breyel, au Pallais, en la gallerie par où on va à la Chancellerie. 
Chevalier, J-C. (1988): «Proverbes et traduction. (La traduction italienne de la Célestine, par Alphonso Ordoñez, Rome, 1506)», BHi XC 1-2, págs. 59-89.

Descimon, R. (1983): Qui étaient les Seize?. Paris, Fédération des sociétés historiques et archéologiques de Paris et de l'île de France.

Descimon, R. y J. J. Ruiz Ibáñez, (2005): Les ligueurs de l'exil. Seysel, Champ Vallon.

Lépinette, B. (2000): «A propos de deux grammaires pour l'enseignement de l'espagnol aux Français: Oudin 1597 et Charpentier 1597». En Bagola, B. (ed.): La lingüistica española en la época de los descubrimientos. Hamburg, H. Buske, págs. 107-119.

L'Etoile, P. de (1741): Journal du regne de Henry IV Roi de France et de Navarre avec des remarques Historiques et Politiques du Chevalier CBA. Et plusieurs Pièces Historiques du même temps, Tome second. La Haye, Chez les Freres Vaillant.

López Alonso, C. y A. Seré: (2000): «La parfaicte méthode (1596). Primer método de español lengua extranjera para franceses ». En Bagola, B. (ed.): La lingüística española en la época de los descubrimientos. Hamburg: H. Buske, págs. 121-135.

Mémoires de la Ligue, contenant les événements les plus remarquables depuis 1576, jusqu'à la Paix accordée entre le Roi de France \& le Roi d'Espagne, en 1598. (1758), Nouvelle édition, revue, corrigée \& augmentée de Notes critiques \& historiques, Tome VI. Amsterdam, chez Arkstée \& Merkens.

Michelet, J. (1980): Euvres complètes. Tome VIII : guerres de religion, la ligue et Henri IV. Paris, Flammarion. Éd. critique par R. Casanova.

Morel-Fatio, A. (1901): Ambrosio de Salazar et l'étude de l'espagnol en France sous Louis XIII. ParisToulouse, Bibliothèque Espagnole.

Moréri, L. (1732): Le Grand Dictionnaire historique ou le mélange curieux de l'histoire sacrée et profane. A Paris, chez Jacques Vincent, rue \& vis-à-vis l'Eglise de Saint Séverin, à l'Ange, MDCCXXXII.

Quijada Van den Berghe, C. (2006): «Datos y criterios para una edición de la obra de Charpentier». En Gómez Asencio, J.J. (dir.): El castellano y su codificación gramatical. De 1492 a 1611. Salamanca, Universidad de Salamanca, págs. 323-334.

Roman d'Amat y M. Prévôt, (1959): Dictionnaire de Biographie française. Paris, Librairie Letouzey et Ané, Tome VIII.

Salazar, A. (1615): Response apolegetique au libelle d'un nommé Oudin. Aux objections duquel sont opposees des responses valables et qui peuvent servir d'instruction en la langue espagnole. Paris, s.n.

Sánchez Pérez, A. (1992): Historia de la enseñanza del español como lengua extranjera (Historiografía de la lingüística española). Madrid, Sociedad General Española de Librería.

Thou, J-A. de (1734): Histoire de Jacque-Auguste de Thou, Depuis 1543 jusqu'en 1607. Traduite sur l'édition latine de Londres. Tome treizième. 1596-1601.

Tuetey, A. (ed) (1902): Histoire Générale de Paris, Registre des délibérations de la Ville de Paris, Tome onzième, 1594-1598. Paris, Imprimerie Nationale. 